\title{
Manifestações orais da doença do enxerto contra o hospedeiro crônica: revisão sistemática de literatura
}

\author{
Oral manifestations of chronic graft vs host disease: systematic literature review
}

\author{
Álvaro Cavalheiro Soares \\ Felipe Souza Lima Alencar \\ Programa de Residência Multiprofissional em Oncologia, Instituto Nacional de Câncer José Alencar Gomes da Silva (INCA), Rio de Janeiro, Brasil \\ Héliton Spindola Antunes \\ Divisão de Ensaios Clínicos e Desenvolvimento de Fármacos, Instituto Nacional de Câncer José Alencar Gomes da Silva (INCA), Rio de Janeiro, Brasil \\ - Os autores declaram que não há conflito de interesse.
}

Resumo

Objetivo:Identificar a frequência das manifestações orais da DECHc em pacientes submetidos ao TCTH, através de uma revisão sistemática da literatura. Material e Métodos: Realizou-se uma revisão sistemática da literatura utilizando-se as bases de dados PubMed, Cochrane e Dentistry \& Oral Sciences Source para realização da busca bibliográfica (setembro/2015), que resultou em um total de 212 publicações, entre as quais 40 foram incluídas no estudo. Resultados: As lesões liquenoides foram relatadas em $82,5 \%$ dos estudos, sendo as alterações mais frequentes entre diversas outras, como úlceras, relatadas em $57,1 \%$ dos estudos, atrofia da mucosa em $45,7 \%$, mucocele em $37,1 \%$ e pseudomembranas em $17,1 \%$. Entre os sintomas, a xerostomia foi relatada em $80 \%$ dos estudos, superando a dor (48,5\%), sensibilidadel queimação (34,2\%) disgeusia/ageusia (17,1\%), disfagia (14,2\%), entre outras. Conclusão: Observou-se assim, na literatura, uma alta ocorrência de diversas manifestações orais associadas à DECHc.

Palavras-chave: transplante de células-tronco; doença enxerto-hospedeiro; erupções liquenoides; úlcera; xerostomia.

\section{Abstract}

Objective: To identify the frequency of oral manifestations of cGVHD in patients undergoing HSCT, through a systematic review of the literature. Material and Methods: It was performed a systematic review of the literature using the databases PubMed, Cochrane and Dentistry \& Oral Sciences Source to perform the literature search (september/2015), which resulted in a total of 212 publications, among which 40 were included in the study. Results: The lichenoid lesions were reported in $82.5 \%$ of the studies, the most frequent changes among many others found, such as ulcers, reported in $57,1 \%$ of the studies, mucosal atrophy in $45,7 \%$, mucocele in $37,1 \%$ pseudomembranes in $17.1 \%$. Among the symptoms, dry mouth was reported in $80 \%$ of studies, overcoming the pain (48,5\%), sensitivity/burning (34,2\%) dysgeusia/ageusia (17,1\%), dysphagia (14,2\%), among others. Conclusion: There was, in the literature, a high occurrence of various oral manifestations associated with cGVHD.

Keywords: stem cell transplantation; graft vs host disease; lichenoid eruptions; ulcer; xerostomia.

\section{Introdução}

A doença do enxerto contra o hospedeiro $(\mathrm{DECH})$ é uma reação imunológica resultante do enxerto de células imunocompetentes de um doador para um hospedeiro imunocomprometido. Ocorre entre $30 \%$ e $70 \%$ dos pacientes submetidos ao transplante de células tronco hematopoiéticas (TCTH) e é a principal causa de morbi-mortalidade a longo prazo. ${ }^{1}$ Embora a fisiopatologia da $\mathrm{DECH}$ não esteja totalmente esclarecida, acredita-se que seja uma doença primariamente mediada por células $\mathrm{T}$ onde há o reconhecimento dos tecidos do paciente como antígeno, devido a diferenças de histocompatibilidade. ${ }^{2}$ Pode ser classificada em aguda ou crônica de acordo com as características clínicas e sinais patológicos das doença, independente do tempo de ocorrência após o TCTH. ${ }^{1,3}$

As manifestações clínicas da DECH afetam mais frequentemente a pele (poiquilodermia, lesões liquenoides, alterações de esclerose, lesões máculo-papulosas), fígado (ALT, AST, bilirrubina total e fosfatase alcalina elevadas - 2x ou mais acima do limite normal), trato gastrintestinal (estreitamento ou estenose do terço superior e médio do esôfago, náusea, vômitos, diarreia) e mucosa oral ${ }^{4}$ e podem estar relacionadas à forma aguda (DECHa) ou crônica (DECHc) da doença. ${ }^{1,3}$

Conforme o National Institutes of Health (NIH), as manifestações orais da DECHc podem ser divididas em: diagnósticas, representadas pelas lesões liquenóides (Figura 1 A); distintivas, representadas por xerostomia, mucoceles (Figura 1 B), atrofia da mucosa, pseudomembranas e úlceras; e alterações comuns à forma aguda da DECH, consistindo em gengivite, mucosite (Figura $1 \mathrm{C}$ ), eritema e dor, sendo por isso auxiliares importantes no diagnóstico dessa condição clínica. ${ }^{1,5}$

As lesões orais podem persistir mesmo após a resolução da DECHc em outros órgãos. O controle de tais lesões, principalmente as ulceradas, é fundamental visto que as mesmas podem servir como porta de entrada para que micro-organismos presentes na microbiota oral alcancem a corrente sanguínea, aumentando o risco de septicemia. ${ }^{6}$ As glândulas salivares também são importantes alvos da DECHc. O diagnóstico precoce da disfunção das glândulas salivares é importante para o manejo das complicações decorrentes da diminuição do fluxo salivar, além de servir como auxílio para a investigação da ocorrência de DECHc em outros órgãos. ${ }^{7-10}$ Outro ponto importante é o risco aumentado que os pacientes com DECHc apresentam de desenvolver uma segunda neoplasia, inclusive o carcinoma de células escamosas de mucosa oral. ${ }^{11}$

Percebe-se, então, que as alterações orais estão frequentemente associadas aos casos de DECHc, sendo consideradas como causas importantes de morbidade e perda de qualidade de vida de sobreviventes a longo prazo, demonstrando a importância da equipe de saúde bucal no suporte a esses indivíduos.

Desta forma, objetivo deste estudo é realizar uma revisão sistemática da literatura, para identificar o perfil das manifestações orais relacionadas à DECHc, considerando os seguintes aspectos: frequência, localização e sintomas clínicos. 

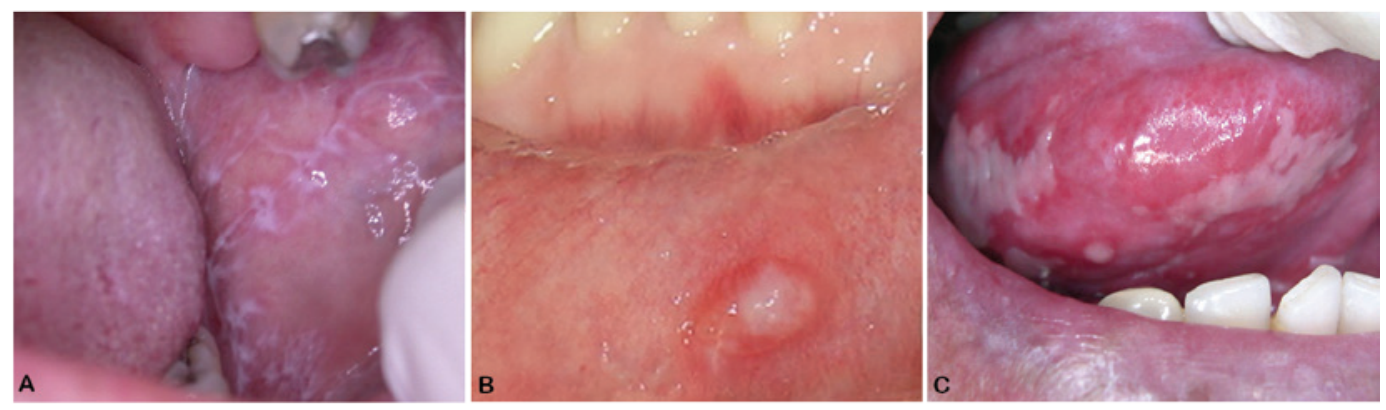

Figura 1. Aspectos clínicos das principais lesões encontradas em pacientes portadores de DECHc: A. Lesão liquenoide localizada em mucosa jugal; B. Mucocele localizada em lábio inferior; $C$. Mucosite localizada em borda lateral de língua

\section{Material e Métodos}

O estudo consiste em uma revisão sistemática da literatura sobre as manifestações orais da DECHc, realizada conformeas etapas a seguir:

1) Elaboração das estratégias de busca: foram utilizadas as bases de dados PubMed, Cochrane e Dentistry \& Oral Sciences Source para a obtenção das produções científicas. As buscas bibliográficas foram realizadas em setembro de 2015, nas bases citadas anteriormente, utilizando-se para isso a combinação dos seguintes descritores de acordo com o DeCS (Descritores em Ciências da Saúde): GVHD, Graft vs Host Disease, Oral Manifestations, Dentists, Mouth e Bucal, com direcionamento para a localização da tais termos nos títulos, resumos e MeSH das publicações, o que resultou nas estratégias de busca e número de publicações, conforme exposto na tabela 1. Não foi estabelecido um intervalo temporal, com o intuito de se obter o maior número de publicações possíveis.

2) Seleção de estudos: para a seleção inicial, as publicações encontradas tiveram suas referências exportadas para o gerenciador de referências Endnote web, para a remoção de duplicações.

3) Critérios de inclusão e exclusão: as publicações foram selecionadas, num primeiro momento, de acordo com a presença ou não de relação com o tema desta pesquisa, através da leitura dos títulos e resumos, de acordo com a análise de um avaliador com experiência no assunto abordado. Foram estabelecidos como critérios de exclusão os estudos em animais, aqueles que citavam apenas alterações associadas à DECH aguda ou ao regime de condicionamento do TCTH e publicações em idiomas diferentes do português, inglês ou espanhol.

4) Análise de dados: dos textos selecionados foram extraídas as informações a respeito das manifestações orais, localização das lesões e sintomas clínicos associados com a DECHc em mucosa oral. As mesmas foram quantificadas, tiveram suas frequências calculadas e estão expressas em tabelas elaboradas com o auxílio do software Microsoft Word 2007 (Microsoft Corporation, Redmond, WA).

Tabela 1. Estratégias de busca nas bases de dados Pubmed, Dentistry \& Oral Sciences Source e Cochrane e número de publicações encontradas

\begin{tabular}{|c|c|c|c|c|}
\hline Base de dados & \multicolumn{3}{|c|}{ Estratégia de busca } & \multirow{2}{*}{$\begin{array}{l}\text { Número de } \\
\text { publicações }\end{array}$} \\
\hline PubMed & \multicolumn{3}{|c|}{$\begin{array}{l}\text { ((GVHD[tiab] OR Graft vs Host Disease[tiab] OR Graft vs Host } \\
\text { Disease[mh]) AND } \\
\text { (Oral Manifestations[tiab] OR Oral Manifestations[mh] OR } \\
\text { Dentist[tiab] OR Dentists[mh] OR Mouth[tiab] OR bucal[tiab])) }\end{array}$} & \\
\hline $\begin{array}{l}\text { Dentistry \& Oral } \\
\text { Sciences Source }\end{array}$ & \multicolumn{3}{|c|}{$\begin{array}{l}\text { (GVHD OR Graft vs Host Disease OR Graft vs Host Disease) AND } \\
\text { (Oral Manifestations OR Oral Manifestations OR Dentistry OR Den- } \\
\text { tists OR Mouth OR bucal) }\end{array}$} & 49 \\
\hline \multirow{9}{*}{ Cochrane } & ID & Search & Hits & \multirow{9}{*}{41} \\
\hline & $\# 1$ & GVHD or Graft vs Host Disease & 892 & \\
\hline & \#2 & MeSH descriptor: [Graft vs Host Disease] this term only & 440 & \\
\hline & \#3 & \#1 or \#2 & 892 & \\
\hline & $\# 4$ & Oral Manifestations or Dentist* or Mouth or bucal & 20307 & \\
\hline & \#5 & MeSH descriptor: [Oral Manifestations] this term only & 2 & \\
\hline & \#6 & MeSH descriptor: [Dentists] this term only & 68 & \\
\hline & \#7 & $\# 4$ or \#5 or \#6 & 20307 & \\
\hline & $\# 8$ & $\# 3$ and \#7 & & \\
\hline
\end{tabular}




\section{Resultados}

A busca resultou em um total de 212 publicações, das quais após a leitura dos títulos, 98 foram selecionadas; destas, 56 foram escolhidas para que seus textos fossem lidos na íntegra (Figura 2 - fluxograma de seleção de estudos de acordo com o PRISMA). Deste total, 16 publicações foram excluídas visto que não foi possível a obtenção do texto completo, nem a realização de contato com os autores das mesmas, o que resultou na leitura de 40 publicações. Das 40 publicações, mais 5 foram excluídas devido aos motivos expostos na figura 2. Sendo assim, 35 publicações tiveram seus textos lidos na íntegra, de onde foram extraídos os dados utilizados neste estudo.

As 35 publicações compreendem a literatura entre 1980 e 2015. Em relação aos tipos de desenho de estudo, a maioria consisteem estudos transversais ou de prevalência (Figura 2). Os resultados encontrados referentes às alterações orais, à localização das lesões e aos sintomas associados estão expressos nas tabelas 2, 3 e 4, respectivamente.

A maior frequência encontrada em relação às alterações orais foi a de lesões liquenóides, relatada em $82,5 \%$ dos estudos. Em contrapartida, alterações como máculas ictéricas, leucoplasia pilosa, pigmentação melanótica, púrpura trombocitopênica, lesões tipo lúpus, escorbuto, celulite, lesões penfigóides, lesões erosivas e diminuição da mobilidade da língua foram descritas somente uma vez, representando, cada uma delas, $2,8 \%$ do total.

Quanto à localização das lesões, 12 estudos não apresentavam tais informações. Diante disso, somente 23 publicações foram utilizadas para obtenção dos dados. As lesões liquenoides apresentaram como principal localização a mucosa jugal, descrita em $69,6 \%$ dos estudos, a segunda localização mais frequente foi o lábio, relatada em $52,2 \%$, seguida da língua, ocorrendo em $39,1 \%$. O eritema teve como localização preferencial a mucosa jugal, visto em $34,8 \%$ dos estudos, e as úlceras ocorreram principalmente em mucosa jugal e língua, ambas com 30,4\%.

Em relação aos sintomas, a xerostomia foi a queixa mais frequente, descrita em $80 \%$ dos estudos, seguida da dor, presente em $48,5 \%$ dos estudos. No outro extremo da tabela encontram-se a sensação de boca e língua enrijecidas, a sensibilidade dentária e a sede frequente, representando $2,8 \%$ do total cada uma delas.

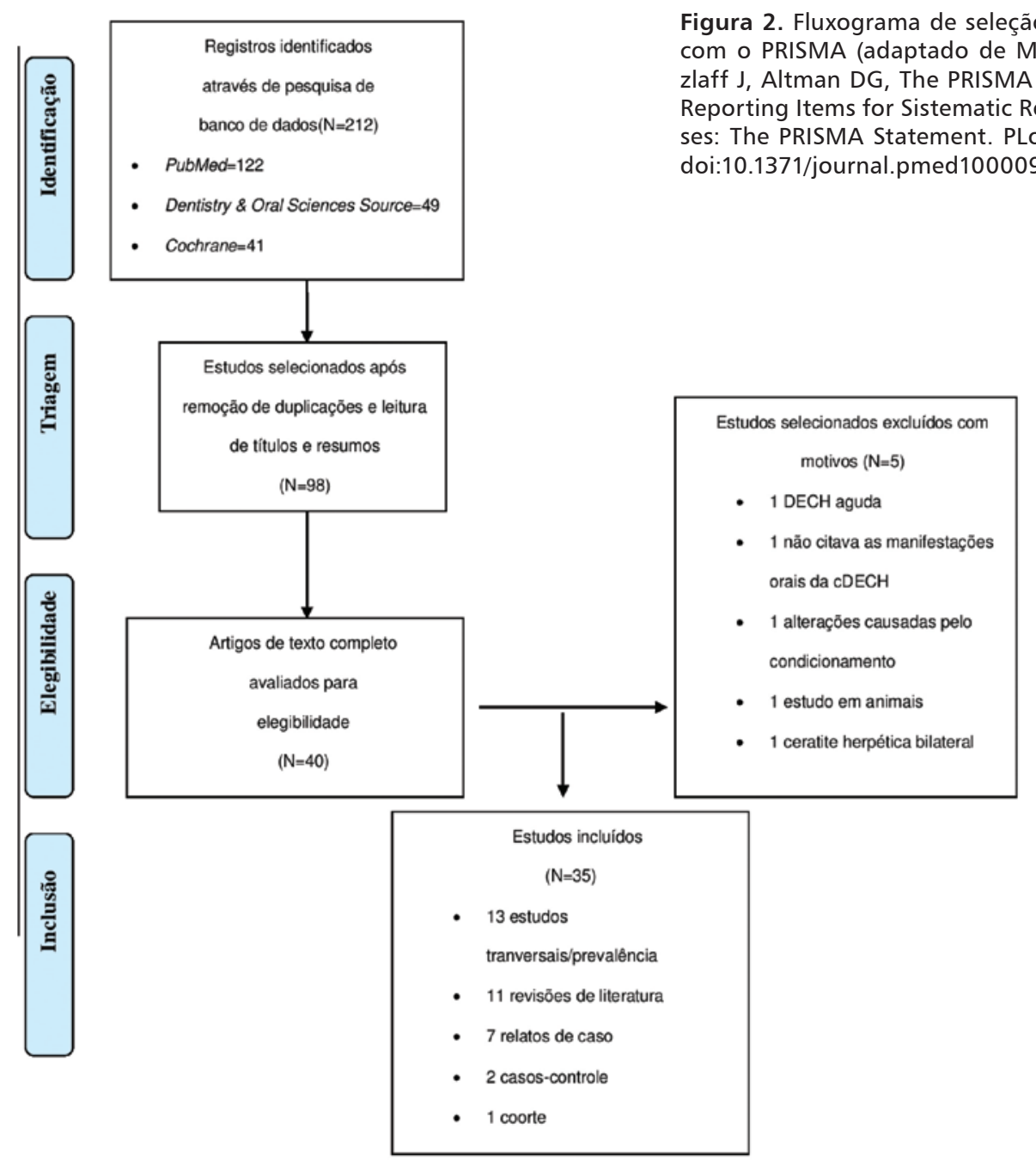


Tabela 2. Frequência das manifestações orais da DECHc relatadas na literatura

\begin{tabular}{|l|c|c|}
\hline \multicolumn{1}{|c|}{ Manifestação oral } & $\mathbf{N}=35$ & $\%$ \\
\hline Lesões liquenoides & 29 & $(82,5 \%)$ \\
\hline Hipossalivação & 28 & $(80 \%)$ \\
\hline Fibrose perioral/Redução da abertura de boca & 20 & $(57,1 \%)$ \\
\hline Eritema & 20 & $(57,1 \%)$ \\
\hline Úlceras & 20 & $(57,1 \%)$ \\
\hline Atrofia da mucosa & 16 & $(45,7 \%)$ \\
\hline Mucoceles & 13 & $(37,1 \%)$ \\
\hline Neoplasias malignas (CEC) & 10 & $(28,5 \%)$ \\
\hline Mucosite & 8 & $(22,8 \%)$ \\
\hline Atrofia das papilas/Glossite atrófica & 6 & $(17,1 \%)$ \\
\hline Pseudomembranas & 6 & $(17,1 \%)$ \\
\hline Placas hiperceratóticas & 6 & $(17,1 \%)$ \\
\hline Leucoplasia & 5 & $(14,2 \%)$ \\
\hline Granuloma piogênico & 5 & $(14,2 \%)$ \\
\hline Alterações gengivais & 4 & $(11,4 \%)$ \\
\hline Xantoma verruciforme & 3 & $(8,5 \%)$ \\
\hline Fibrose dos tecidos moles & 2 & $(5,7 \%)$ \\
\hline Edema da mucosa & 2 & $(5,7 \%)$ \\
\hline Queilite angular & 2 & $(5,7 \%)$ \\
\hline Máculas ictéricas & 1 & $(2,8 \%)$ \\
\hline Leucoplasia pilosa & 1 & $(2,8 \%)$ \\
\hline Pigmentação melanótica & 1 & $(2,8 \%)$ \\
\hline Púrpura trombocitopênica & 1 & $(2,8 \%)$ \\
\hline Lesões tipo lúpus & 1 & $(2,8 \%)$ \\
\hline Escorbuto & 1 & $(2,8 \%)$ \\
\hline Celulite & 1 & $(2,8 \%)$ \\
\hline Lesões penfigoides & 1 & $(2,8 \%)$ \\
\hline Lesões erosivas & 1 & $(2,8 \%)$ \\
\hline Diminuição da mobilidade da língua & 1 & $(2,8 \%)$ \\
\hline & & \\
\hline
\end{tabular}

Tabela 3. Localização das lesões orais associados à $\mathrm{DECHc}$ relatadas na literatura $(\mathrm{N}=23)$

\begin{tabular}{|l|c|c|c|c|c|c|c|}
\hline \multicolumn{1}{|c|}{ Localização } & Mucosa jugal & Lábio & Língua & Palato & Assoalho & Gengiva & Trígono retromolar \\
\hline Lesões liquenoides & $16(69,6 \%)$ & $12(52,2 \%)$ & $9(39,1 \%)$ & $4(17,4 \%)$ & $2(8,7 \%)$ & $4(17,4 \%)$ & $1(4,3 \%)$ \\
\hline Eritema & $8(34,8 \%)$ & $6(26 \%)$ & $4(17,4 \%)$ & $2(8,7 \%)$ & $2(8,7 \%)$ & $2(8,7 \%)$ & $0(0 \%)$ \\
\hline Úlceras & $7(30,4 \%)$ & $4(17,4 \%)$ & $7(30,4 \%)$ & $6(26 \%)$ & $2(8,7 \%)$ & $3(13 \%)$ & $0(0 \%)$ \\
\hline Mucoceles & $0(0 \%)$ & $4(17,4 \%)$ & $0(0 \%)$ & $5(21,7 \%)$ & $0(0 \%)$ & $0(0 \%)$ & $0(0 \%)$ \\
\hline Neoplasias malignas (CEC) & $0(0 \%)$ & $0(0 \%)$ & $1(4,3 \%)$ & $1(4,3 \%)$ & $0(0 \%)$ & $1(4,3 \%)$ & $1(4,3 \%)$ \\
\hline Mucosite & $0(0 \%)$ & $1(4,3 \%)$ & $0(0 \%)$ & $0(0 \%)$ & $0(0 \%)$ & $0(0 \%)$ & $0(0 \%)$ \\
\hline Pseudomembranas & $1(4,3 \%)$ & $0(0 \%)$ & $1(4,3 \%)$ & $0(0 \%)$ & $0(0 \%)$ & $0(0 \%)$ & $0(0 \%)$ \\
\hline Placas hiperceratóticas & $2(8,7 \%)$ & $1(4,3 \%)$ & $2(8,7 \%)$ & $1(4,3 \%)$ & $0(0 \%)$ & $1(4,3 \%)$ & $0(0 \%)$ \\
\hline Leucoplasia & $0(0 \%)$ & $0(0 \%)$ & $1(4,3 \%)$ & $0(0 \%)$ & $0(0 \%)$ & $0(0 \%)$ & $0(0 \%)$ \\
\hline Granuloma piogênico & $0(0 \%)$ & $0(0 \%)$ & $2(8,7 \%)$ & $0(0 \%)$ & $0(0 \%)$ & $0(0 \%)$ & $0(0 \%)$ \\
\hline Xantoma verruciforme & $0(0 \%)$ & $0(0 \%)$ & $1(4,3 \%)$ & $0(0 \%)$ & $0(0 \%)$ & $0(0 \%)$ & $0(0 \%)$ \\
\hline Pigmentação melanótica & $0(0 \%)$ & $1(4,3 \%)$ & $0(0 \%)$ & $0(0 \%)$ & $0(0 \%)$ & $0(0 \%)$ & $0(0 \%)$ \\
\hline Púrpura trombocitopênica & $0(0 \%)$ & $0(0 \%)$ & $0(0 \%)$ & $0(0 \%)$ & $1(4,3 \%)$ & $0(0 \%)$ & $0(0 \%)$ \\
\hline Lesões tipo lúpus & $0(0 \%)$ & $0(0 \%)$ & $0(0 \%)$ & $1(4,3 \%)$ & $0(0 \%)$ & $0(0 \%)$ & $0(0 \%)$ \\
\hline
\end{tabular}


Tabela 4. Frequência dos sintomas orais associados à $\mathrm{DECH}$ relatados na literatura

\begin{tabular}{|l|c|c|}
\multicolumn{1}{|c|}{ Sintomas } & N=35 & $\%$ \\
\hline Xerostomia & 28 & $(80 \%)$ \\
\hline Dor & 17 & $(48,5 \%)$ \\
\hline Sensibilidade/Queimação & 12 & $(34,2 \%)$ \\
\hline Disgeusia/Ageusia & 6 & $(17,1 \%)$ \\
\hline Disfagia & 5 & $(14,2 \%)$ \\
\hline Dislalia & 3 & $(8,5 \%)$ \\
\hline Boca e língua enrijecidas & 1 & $(2,8 \%)$ \\
\hline Sensibilidade dentária & 1 & $(2,8 \%)$ \\
\hline Sede frequente & 1 & $(2,8 \%)$ \\
\hline
\end{tabular}

\section{Discussão}

A DECHc é uma patologia complexa que proporciona grandes complicações à vida dos pacientes submetidos ao TCTH. Diante disso, a realização de um diagnóstico precoce e preciso mostra-se extremamente importante para o estabelecimento de um tratamento eficaz em estágios iniciais da doença. Enquanto manifestações em outros órgãos, como a pele e o fígado, por exemplo, podem ser confundidas com sinais de outras doenças; determinadas manifestações orais, como as lesões liquenoides, podem ser muito úteis no auxílio ao diagnóstico, visto que o envolvimento oral tem sido descrito como uma das alterações iniciais da DECHc. ${ }^{2}$

De acordo com os resultados deste estudo, pode-se observar a alta frequência, na literatura, das lesões liquenoides, relatadas em $82,5 \%$ das publicações. A presença de tais lesões em pacientes submetidos ao TCTH foi estabelecida pelo consensos de 2005 e 2014 do NIH como critério clínico suficiente, independentemente da realização de exame histopatológico, para o diagnóstico da DECHc. ${ }^{1,3}$ Tal decisão é fortemente baseada na grande correlação clínica entre tal lesão e a $\mathrm{DECHc} \mathrm{e} \mathrm{oamplo} \mathrm{relato} \mathrm{na} \mathrm{literatura} \mathrm{desta} \mathrm{associação,} \mathrm{como} \mathrm{em} \mathrm{diversos} \mathrm{artigos} \mathrm{utilizados} \mathrm{nesta} \mathrm{revisão} \mathrm{e} \mathrm{os} \mathrm{próprios}$ resultados encontrados. A fibrose perioral/redução da abertura de boca e as placas hiperceratóticas relatadas em 57,1\% e 17,1\% dos estudos, respectivamente, deixaram de ser consideradas como alterações diagnósticas orais de acordo com o último consenso do NIH. ${ }^{1}$

Em relação às alterações distintivas: úlceras (57,1\%), atrofia da mucosa $(45,7 \%)$ e mucocele $(37,1 \%)$ foram a terceira, sexta e sétima alterações mais relatadas, mostrando assim alta associação com a DECHc.

Fato relevante foi a alta frequência dos relatos de eritema $(57,1 \%)$ e dor $(48,5 \%)$, sendo a $3^{\text {a }}$ manifestação e o $2^{\circ}$ sintoma, mais frequentes na literatura, sendo ambas consideradas alterações comuns à forma aguda e crônica da DECH.

Um dado importante mostra-se em relação à ocorrência de neoplasias malignas (principalmente o Carcinoma de Células Escamosas), representando 28,5\% das alterações encontradas neste estudo (oitava mais frequente). Fato este que deve ser levado em consideração haja vista que trata-se de uma doença agressiva que influência de forma importante tanto na qualidade de vida assim como na sobrevida global do paciente, sendo descrito na literatura como possível alteração oral associada à DECHc. ${ }^{13}$ Entre os fatores de risco propostos para explicar esta alta incidência, encontram-se o processo inflamatório associado à DECHc, a imunossupressão prolongada devido ao tratamento da DECHc, disfunção imunológica, a anemia de Fanconi, mutações induzidas pela radiação e os efeitos carcinogênicos e citotóxicos de diversas medicações..$^{14}$ Portanto, ainda não está totalmente claro se é a DECHc em si, ou se é o seu tratamento que está mais fortemente associado ao risco de desenvolvimento de tal neoplasia, ${ }^{15}$ demonstrando, desta maneira, a necessidade de maiores investigações a respeito do assunto.

As demais alterações (leucoplasia, granuloma piogênico, alterações gengivais, xantoma verruciforme, entre outras) foram pouco relatadas (frequência $<15 \%$ ) sendo provavelmente achados.

Excetuando-se as pseudomembranas, todas as outras cinco alterações, diagnósticas ou distintivas, estão entre as sete mais frequentemente relatadas na literatura. Sendo assim, os dados corroboram com o consenso do NIH em relação às principais alterações orais para diagnóstico da DECHc.

No que diz respeito à localização das lesões, pode-se observar que nem todos os estudos descreveram o local de acometimento das mesmas, e os que descreveram, na sua grande maioria, não citavam a localização intraoral de todas as lesões. $\mathrm{O}$ conhecimento das localizações das lesões, por exemplo, a alta ocorrência de lesões liquenoides em mucosa jugal e em lábio, pode auxiliar o profissional de saúde a direcionar o exame intraoral para regiões mais específicas com o intuito da realização de um diagnóstico mais preciso. Além disso, a ocorrência de determinados tipos de lesões dolorosa, como as úlceras em palato e em gengiva, descritas em $26 \%$ e $13 \%$ dos estudos, respectivamente, dificultam a realização de uma adequada higienização oral, predispondo o indivíduo ao desenvolvimento de cáries e doença periodontal.

Em relação aos sintomas, a xerostomia foi a queixa mais frequente, sendo descrita em $80 \%$ dos estudos, superando a dor, descrita em $48,5 \%$ dos estudos ( $2^{\text {a }}$ mais frequente) e a sensibilidade/queimação presente em $34,2 \%$ dos estudos. A ocorrência 
elevada de hipossalivação/xerostomia é um dado que corrobora com a hipótese do envolvimento das glândulas salivares na DECHc. Apesar de muito frequente, nenhum dos estudos deixou claro as medicações em uso pelos pacientes e a idade dos mesmos, fatores estes que influenciam na quantidade e qualidade da saliva dos indivíduos. Embora poucos estudos tenham relatado os métodos utilizados para o diagnóstico de hipossalivação, vários deles demonstraram o comprometimento das glândulas salivares pela DECHc. ${ }^{16,17}$

De forma geral os resultados encontrados auxiliam na elaboração de um perfil das principais manifestações orais associadas à DECHc. Todavia, a presente revisão revela um baixo número de estudos na literatura a respeito do assunto; sendo este o motivo da inclusão de todas as 35 publicações selecionadas, independentementedos tipos de desenho de estudo (revisões de literatura, relatos de caso, estudos tranversais/prevalência, casos-controle e coorte). Portanto, mostra-se necessária a realização de novas pesquisas para o maior conhecimento da DECHc oral, uma vez que a identificação das manifestações orais é de relevante valor para a realização de um diagnóstico precoce, proporcionando o estabelecimento de uma terapêutica em estados iniciais da doença, o que aumenta sua eficácia e consequentemente melhora a qualidade de vida dos pacientes portadores de tal condição.

\section{Conclusão}

A presente revisão sistemática tornou evidente a relação entre diversas alterações orais e a DECHc. Destaca-se a marcante frequência das lesões liquenoides, o que ressalta seu grande valor no diagnóstico de tal condição. Isso corrobora com os critérios diagnósticos definidos no consenso do NIH, reafirmando sua importância na prática clínica. Sendo assim, esta revisão auxiliou na identificação do perfil, assim como, sintetizou o conhecimento adquirido ao longo dos anos em relação às manifestações orais daDECHc. 
1. Jagasia MH, Greinix HT, Arora M, Williams KM, Wolff D, Cowen EW, et al. National Institutes of Health consensus development project on criteria for clinical trials in chronic graft-versus-host disease: I. The 2014 Diagnosis and Staging Working Group Report. Biol Blood Marrow Transplant. 2015;21:389-401.

2. Busca A, Locatelli F, Vai S, Dall'Omo AM, Gar-giulo A, Falda M. Clinical grading of oral chronic graft-versus-host disease in 104 consecutive adult patients. Haematologica. 2005;90(4):567-9.

3. Filipovich AH, Weisdorf D, Pavletic S, Socie G, Wingard JR, Lee SJ, et al. National Institutes of Health consensus development project on criteria for clinical trials in chronic graft-versus-host disease: I. Diagnosis and Staging Working Group Report. Biol Blood Marrow Transplant. 2005;11(12):945-56.

4. Ratanatharathorn V, Ayash L, Lazarus HM, FU J, Uberti JP. Chronic graft-versus-host disease: cliniาcal manifestation and therapy. Mini review. Bone Marrow Transplant. 2001;28:121-9.

5. Lee SJ, Wolff D, Kitko C, Koreth J, Inamoto Y, Jagasia M, et al.Measuring therapeutic response in chronic graft-versus-host disease. National Institutes of Health consensus development project on criteria for clinical trials in chronic graft-versus-host disease: IV. The 2014 Response Criteria Working Group. Biol Blood Marrow Transplant. 2015;21:984-99.

6. Miranda FC, Domínguez-Martins M, Volpe A, Pallota R, Araujo NS. Severe oral manifestations of chronic graft-vs-host disease. JADA. 2001;132:1124-7.

7. Nagler RM, Nagler A. Pilocarpine hydrochloride relieves xerostomia in chronic graft-versus-host disease: a sialometrical study. Bone Marrow Transplantation.1999;23(10):1007-11.

8. Nagler RM, Nagler A. The effect of pilocarpine on salivary constituents in patients with chronic graft-versus-host disease. Arch Oral Biol.2001;46(8):689-95.

9. Nagler RM, Nagler A. Sialometrical and sialochemical analysis of patients with chronic graft-versus-host disease - a prolonged study. Cancer Invest.2003;21(1):34-40.

10. Singhal S, Powles R, Treleaven J, Rattenbury H, Mehta J. Pilocarpine hydrochloride for symptomatic relief of xerostomia due to chronic graft-versus-host disease or total-body irradiation after bone-marrow transplantation for hematologic malignancies.Leuk Lymphoma. 1997;24(56):539-43

11. Meier JKH, Wolff D, Pavletic S, Greinix H, Gosau M, Bertz H, et al. Oral chronic graft-versus-host disease: report from the International Consensus Conference on clinical practice in cGVHD Clin Oral Invest. 2010;15(2):127-39.

12. Resende RG, Correia-Silva JF, Arão TC, Brito JAR, Bittencourt H, Gomez RS, et al. Oral cGVHD screening tests in the diagnosis of systemic chronic graft-versus-host disease. Clin Oral Invest. 2011;16(2):565-70.

13. Petti S, Polimeni A, Berloco PB, Scully C. Orofacial diseases in solid organ and hematopoietic stem cell transplant recipients. Oral Dis. 2012;19(1):18-36.

14. Mays JW, Fassil H, Edwards DA, Pavletic SZ, Bassim CW. Oral chronic graft-versus-host disease: current pathogenesis, therapy, and research. Oral Dis. 2012;19:327-46.

15. Imanguli MM, Alevizos I, Brown R, Pavletic SZ, Atkinson JC. Oral graft-versus-host disease. Oral Diseases. 2008;14(5):396-412.

16. Shulman HM, Cardona DM, Greenson JK, Hingorani S, Horn T, Huber E, et al. NIH Consensus development project on criteria for clinical trials in chronic graft-versus-host disease: II. The 2014 Pathology Working Group Report.Biol Blood Marrow Transplant. 2015;21(4):589-603.

17. Coracin FL, Pizzigatti Correa ME, Camargo EE, Peterson DE, de Oliveira Santos A, Vigorito AC, et al. Major salivary gland damage in allogeneic hematopoietic progenitor cell transplantation assessed by scintigraphic methods.Bone Marrow Transplant. 2006;37(10):955-9.

18. Filipovich, AH. Diagnosis and manifestations of chronic graft-versus-host disease. Best Practice \& Research Clinical Haematology. 2008;21(2):251-7.
19. Treister NS, Cook JrEFC, Antin J, Lee SJ, Soiffer R, Woo SB. Clinical evaluation of oral chronic graft-versus-host disease. Biology Of Blood And Marrow Transplant.2008;14(1):110-5.

20. Woo SB, Lee SJ, Schubert MM. Graft-vs.-host disease. Critical Reviews In Oral Biology \& Medicine.1997;8(2):201-16.

21. Treister NS, Woo SB, O'Holleran EW, Lehmann LE, Parson SK, Guinan EC. Oral chronic graft-versus-host disease in pediatric patients after hematopoietic stem cell transplantation. Biology Of Blood And Marrow Transplantation. 2005;11(9):721-31.

22. Cavalcanti LG, Araújo RLF, Bonfim C, Torres-Pereira CC. Oral manifestations compatible with chronic graft-versus-host disease in patients with fanconi anemia. Biology OfBlood And Marrow Transplantation.2015;21(2):275-80.

23. Torres-Pereira CC, Stramandinoli-Zanicotti RT, Amenábar JM, Sassi LM, Pedruzzi PAG, Piazetta CM, Bonfim C. Oral squamous cell carcinoma in two siblings with Fanconi anemia after allogeneic bone marrow transplantation. Spec Care Dentist. 2013;34(4):212-5.

24. Brand HS, Bots CP, Raber-Durlacher JE. Xerostomia and chronic oral complications among patients treated with haematopoietic stem cell transplantation. British Dental Journal. 2009;207(9):1-4.

25. Bassim CW, Fassil H, Mays JW, Edwards D, BairdK, Steinberg SM, et al. Oral disease profiles in chronic graft versus host disease. Journal Of Dental Research.2015; 94(4):547-54.

26. Kletzel M, Powers K, Hayes M. Scurvy: A new problem for patients with chronic GVHD involving mucous membranes; an easy problem to resolve. Pediatr Transplantation. 2014;18(5):524-26.

27. Castellarin P, Stevenson K, Biasotto M, Yuan A, Woo SK, Treister NS. Extensive Dental Caries in Patients with Oral chronic graft-versus-host disease. Biology Of Blood And Marrow Transplantation.2012;18(10):1573-9.

28. Horwitz ME, Sullivan KM. Chronic graft-versus-host disease. Blood Reviews. 2006;20(1):15-27.

29. Majorana A, Schubert MM, Porta F, Ugazio AG, Sapelli PL. Oral complications of pediatric hematopoietic cell transplantation: diagnosis and management. Support Care Cancer.2000;8:353-65.

30. Bassim CW, Fassil H, Mays JW, Edwards D, Baird K, Steinberg SM, et al. Validation of the National Institutes of Health chronic GVHD oral mucosal score using component-specific measures.Bone Marrow Transplant.2014;49:116-21.

31. Lima ENA, Fernandes MA, Ferreira MAF, Nonaka CFW, Freitas MA, Medeiros AMC. Bone marrow transplantation: graft-versus-host disease and oral changes. Rev Odonto Cienc. 2012;27(1):10-5.

32. Shulman HM, Sullivan KM, Weiden PL, Mcdonald GB, Striker GE, Sale GE, et al. Chronic graft-versus-host disease syndrome in man: a long-term clinicopathologic study of 20 Seattle patients. The American Journal of Medicine. 1980;69:204-17.

33. Nappali D, Lingappa A. Oral manifestations in transplant patients. Dent Res J. 2015;12(3):199-2008.

34. Maturo P, Condò R, Costacurta M, Docimo R. Oral manifestations in the chronic graft-versus-host disease in paediatric patients: case report. Oral \& Implantology. 2009;2:34-41.

35. Nicolatou-Galitis O, Kitra V, Vliet-Constantinidou CV, Peristeri J, Goussteis E, Petropoulos D, et al. The oral manifestations of chronic graft-versus-host disease (cGVHD) in paediatric allogenic bone marrow transplant recipients. J Oral Pathol Med. 2001;30:148-53.

36. Eggleston TI, Ziccardi VB, Lumerman H, Newark NJ. Graft-versus-host disease: case report and discussion. Oral Surg Oral Med Oral Pathol Oral Radiol End. 1998;86:696-6.

37. Dahllof G, Heimdahl A, Modéer T, Twetman S, Bolme P, Ringdén O. Oral mucou membrane lesions in children treated with bone marrow transplantation. Scand J Dent Res. 1989;97:268-77.

38. Carl W. Bone marrow transplants and oral complications. Quintessence Int. 1984;10:1001-09.

39. Curtis Jr JW, Caughman GB, Augusta GA. Na apparent unusual re- 
lationship between rampant caries and the oral mucosal manifestations of chronic graft-versus-host disease. Oral Surg Oral Med Oral Pathol. 1994;78:267-72.

40. Mattsson T, Sundqvist KG, Heimdahl A, Dahloff G, Ljungman P, Ringdén $\mathrm{O}$. A comparative immunological analysis of the oral mucosa in chronic graft-versus-host disease and oral lichen planus. Archs Oral Biol. 1992;37(7)539-47.

41. Hiroki A, Nakamura S, Shinohara M, Oka M. Significance of oral examination in chronic graft-versus-host disease. J Oral Pathol Med. 1994;23:209-15.
42. García ER, Molina RB,González MTJV. Graft-versus-host disease, na eight case report and literature review. Med Oral Patol Oral Cir Bucal. 2006;11:486-92.

43. da Fonseca MA, Hong C. An overview of chronic graft-versus-host disease following pediatric hematopoietic stem cell transplantation. Pediatric Dentistry. 2008;30(2):98-104.

44. Lee SJ, Flowers MED. Recognizing and managing chronic graft-versus-host disease. Hematology. 2008;1:134-48.

45. Pereira CM, Melo LG, Gasparetto PF, Corrêa MEP. Chronic graft-versus-host disease: a scleroderma-like case report. RSBO. 2011;8(2):236-9.

Recebido em: 26/04/2016 / Aprovado em: 07/06/2016

\section{Álvaro Cavalheiro Soares}

Rua Dr. Alfredo Backer, 329/bloco 2/apto. 504 - Alcântara

São Gonçalo/RJ, Brasil - CEP: 24452-001

E-mail: alvarosoares90@gmail.com 